\title{
Dynamic Conductance of Carbon Nanotubes
}

\author{
Christopher Roland, ${ }^{1}$ Marco Buongiorno Nardelli, ${ }^{1}$ Jian Wang, ${ }^{2}$ and Hong Guo ${ }^{3}$ \\ ${ }^{1}$ Department of Physics, North Carolina State University, Raleigh, North Carolina 27695 \\ ${ }^{2}$ Department of Physics, The University of Hong Kong, Pokfulam Road, Hong Kong, China \\ ${ }^{3}$ Center for the Physics of Materials and Department of Physics, McGill University, Montreal, Quebec, Canada H3A 2T8
}

(Received 9 September 1999)

\begin{abstract}
The dynamic conductance of carbon nanotubes was investigated using the nonequilibrium Green's function formalism within the context of a tight-binding model. Specifically, we have studied the ac response of tubes of different helicities, both with and without defects, and an electronic heterojunction. Because of the induced displacement currents, the dynamic conductance of the nanotubes differs significantly from the dc conductance displaying both capacitive and inductive responses. The important role of photonassisted transport through nanotubes is revealed and its implications for experiments discussed.
\end{abstract}

PACS numbers: 72.80.Rj, 73.61.Wp

Depending on their helicity, single-wall nanotubes are either metallic or semiconducting [1,2], and therefore have the potential of forming the basis of a future, nanotubebased molecular electronics [2-4]. To explore this exciting possibility, the electronic properties of nanotubes have been the subject of numerous theoretical [1-10] and experimental investigations [11-14]. So far, the nanotubebased electronic devices that have been investigated have relied on the dc response of the nanotubes. It is, however, well known that the ac response of devices is at least equally, and often even more, important for understanding and designing high speed applications. ac transport measurements are of great interest because they provide information about the electrochemical capacitance, the nonequilibrium charge distribution, the dynamic coupling of devices, and the transport dynamics of a conductor. Moreover, the induced charges within a conductor, on a nearby gate, or on the surface of an electron reservoir can all play an important role in determining ac transport. These features all give rise to significant complications in the formulation of transport theory at the nanometer length scale, and are the focus of current, ongoing theoretical research $[15,16]$. In this Letter, we report on a theoretical investigation of the dynamic conductance of single-wall carbon nanotubes, both with and without defects. The resulting dynamic conductance differs substantially from the dc conductance, as the tubes display both capacitive and inductive behavior. We also show that the ac transport through nanotube-based devices is strongly influenced by photon-assisted transmission, and suggest a number of ways in which these predictions may be tested. While the details of our results are specific for nanotubes, we believe that our calculations describe general features of the ac conductance of materials at the nanometer length scale.

While quantum transport under dc condition is well understood [17,18], the ac response of systems is complicated by the presence of time-dependent fields that can take the system out of equilibrium. Under ac conditions, electrodynamics shows that displacement currents are induced, which can substantially alter the transport properties of the system [15]. In order to predict the dynamic conductance of nanoscale conductors such as the carbon nanotubes, the inclusion of these displacement currents into the theoretical formalism is absolutely necessary for two fundamental reasons. First, they are needed if the total current in the system is to be conserved. Second, quantum transport must be gauge invariant, which implies that the physics will depend only on the voltage differences. Another important phenomena associated with the ac response of a conductor is that of photon-assisted tunneling: in the presence of a time-varying potential, electrons can absorb photons, and thereby inelastically tunnel through other levels. Photonassisted transport has been studied experimentally and theoretically in a variety of semiconductor quantum dots, superconducting tunnel junctions, and superlattices [19-21].

The dynamic conductance $g_{\alpha \beta}(\omega)$ as a function of frequency $\omega$ measured between two leads $\alpha, \beta$ is defined through the current $I_{\alpha}: I_{\alpha}=\sum_{\beta} g_{\alpha \beta} V_{\beta}$. Because of current conservation and gauge invariance, the conductance coefficients must satisfy sum rules $\sum_{\beta} g_{\alpha \beta}=0$ and $\sum_{\alpha} g_{\alpha \beta}=0$. To calculate $g_{\alpha \beta}(\omega)$, we have made use of a recently developed formalism in which the conduction and displacement currents are properly partitioned among the leads [22], and which incorporates the electron-electron interactions at the Hartree level [23]. This formalism is based on the Keldysh nonequilibrium Green's function [24] and shows that $g_{\alpha \beta}(\omega)$ is given as the sum of two terms, $g_{\alpha \beta}=g_{\alpha \beta}^{c}+A_{\alpha} g_{\beta}^{d}$. The first term $g_{\alpha \beta}^{c}$ describes the frequency dependent admittance matrix due to conduction current of carriers, while the second term represents the contribution of the displacement current [22]. In the low temperature limit, the first term is given by $g_{\alpha \beta}^{c}(\omega)=\left(2 e^{2} / h\right) \int_{\epsilon}^{\epsilon+\hbar \omega} g_{\alpha \beta}^{c}(\epsilon, \omega) /(\hbar \omega) d \epsilon$, with

$g_{\alpha \beta}^{c}(\epsilon, \omega)=\operatorname{Tr}\left[\bar{G}_{0}^{r} \Gamma_{\beta} G_{0}^{a} \Gamma_{\alpha}\right]-i \delta_{\alpha \beta} \operatorname{Tr}\left[\bar{G}_{0}^{r} \Gamma_{\alpha}-\Gamma_{\alpha} G_{0}^{a}\right]$,

where $G_{0}^{r, a}$ are retarded and advanced Green's function of the nanotube and $\Gamma_{\alpha}$ the coupling between lead $\alpha$, respectively $[18,22]$. Here, we have used the abbreviation 
$G \equiv G(\epsilon)$ and $\bar{G} \equiv G(\epsilon+\hbar \omega)$ for the Green's functions with energy $\epsilon$. This expression is valid in the wideband limit where the couplings of the leads are independent of the energy [25]. The second term of $g_{\alpha \beta}(\omega)$ is defined via $A_{\alpha}=-\sum_{\gamma} g_{\alpha \gamma}^{c} / \sum_{\gamma} g_{\gamma}^{d}, g_{\beta}^{d}(\omega)=\left(2 e^{2} / h\right) \int_{\epsilon}^{\epsilon+\hbar \omega} g_{\beta}^{d} \times$ $(\epsilon, \omega) d \epsilon$, and $g_{\beta}^{d}(\epsilon, \omega)=\operatorname{Tr}\left[\bar{G}_{0}^{r} \Gamma_{\beta} G_{0}^{a}\right]$. This term is especially crucial, as it ensures that the currents within the conductor are conserved. Much of the new physics associated with the ac response of nanotubes derives from this contribution. To numerically calculate the Green's functions, and the couplings $\Gamma_{\alpha}$ between the leads (assumed to be perfect nanotubes) and the device, we have used a now standard tight-binding-based technique $[2,9,10,18]$. The nanotube Hamiltonian was represented by a singleparameter, nearest-neighbor $\pi$-orbital tight-binding model with bond potential $V_{p p \pi}=\gamma_{0}=-2.75 \mathrm{eV}$, which is known to give a reasonable, qualitative description of the electronic and transport properties of carbon nanotubes $[2,9]$.

Before presenting our ac data, we briefly review dc transport in perfect carbon nanotubes [1-10]. Metallic nanotubes are characterized by the crossing of two bands at the Fermi level, so that the theoretical dc conductance in units of $2 e^{2} / h$ has a value of two. Since no other bands are available for the energy range $\epsilon \sim \pm 0.8$ [for a $(10,10)$ tube], the dc conductance is a constant over this energy range. At larger energies, electrons are able to probe different energy bands, giving rise to an increase of dc conductance with the increase proportional to the number of additional bands available for transport. The dc conductance curve therefore consists of a series of "down-and-up" steps as shown in Fig. 1. The step positions correlate with the band edges which are marked by peaks in local density of states (LDOS).

Figure 1 shows the ac conductance of a $(10,10)$ metallic tube at different ac frequencies. Since $g_{12}(\omega)$ is complex, we plot its real (dissipative part) and imaginary parts (nondissipative) separately. We focus on $\hbar \omega \leq 4 \mathrm{eV}$, which is well below the plasma frequency of nanotubes [26]. We also note that in all cases, the expected dc results are smoothly recovered in the limit $\hbar \omega \rightarrow 0$. Indeed, for $0 \leq \hbar \omega \leq$ $0.1 \mathrm{eV}$, the differences between the ac and dc conductances are relatively small. At larger values of $\hbar \omega$ significant differences begin to emerge. Most importantly, for $0.1 \leq \hbar \omega \leq 1 \mathrm{eV}$ there is a general reduction in the value of the ac conductance, along with a gradual loss of the step structure. For larger values of $\hbar \omega$, the ac conductance increases again so that for $2 \mathrm{eV}, g_{12}(\omega)$ not only recovers but is larger than its initial value. This behavior is shown in detail in Fig. 2, which gives a plot of ac conductance versus frequency for two specific energies. Another important feature of ac conductance is the emergence of an imaginary component or "phase," shown at the top of Fig. 1. While initially zero, this imaginary component builds up as the frequency increases, developing an oscillatory pattern that undergoes large variations in both size and sign.

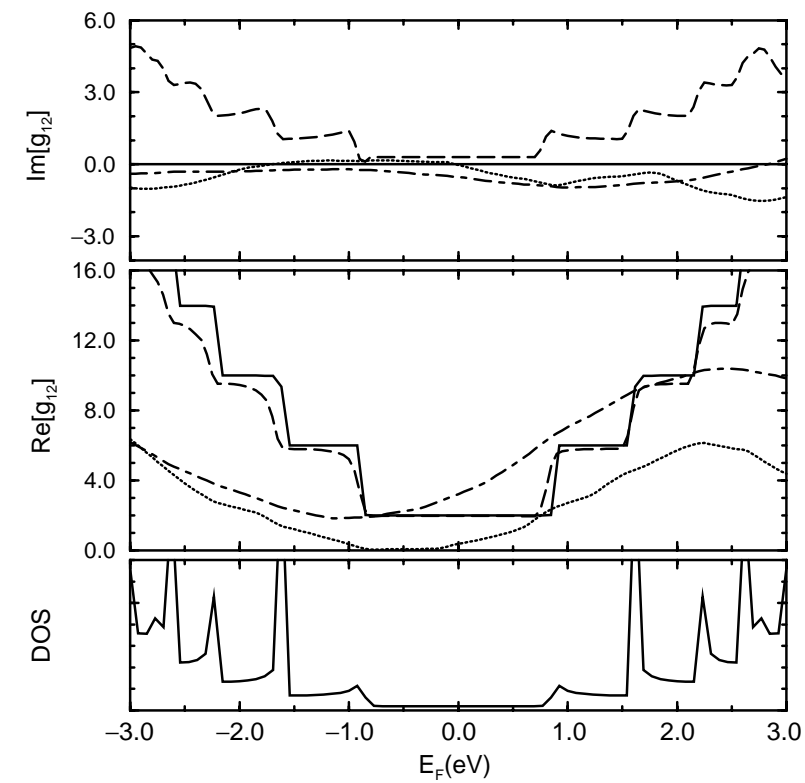

FIG. 1. Real and imaginary parts of $g_{12}(\hbar \omega)$ for a $(10,10)$ metallic tube at different frequencies in units of $2 e^{2} / h$ : solid line, $\hbar \omega=0$; dashed line, $0.1 \mathrm{eV}$; dotted line, $1.0 \mathrm{eV}$; and dot-dashed line, $2.0 \mathrm{eV}$. The DOS (arbitrary units) is shown underneath and marks the band edges.

These results may be understood as follows. The imposition of a time-varying current in the leads induces a displacement current, even inside pristine nanotubes. This displacement current acts to oppose the change of normal conduction current, thereby leading to an overall decrease in the ac conductance as $\omega$ is increased from zero. However, if the frequency is large enough, photon-assisted transport can place the electrons into higher subbands which is signaled by a corresponding increase in ac conductance (Fig. 2). The net result is that $g_{\alpha \beta}(\omega)$, after an initial decrease, is greatly enhanced. ac transport is also characterized by the phase of the current: either current follows voltage in phase which implies an inductive

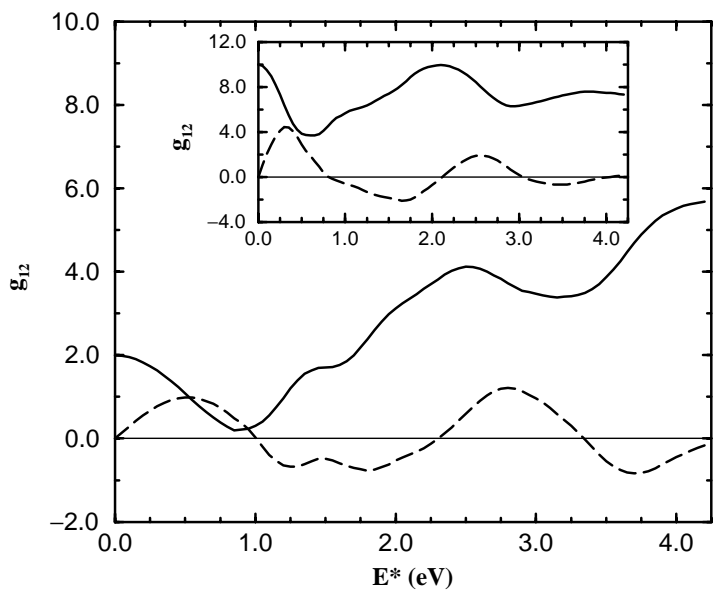

FIG. 2. Real (solid) and imaginary (dashed) components of $g_{12}$ (units $\left.2 e^{2} / h\right)$ for a $(10,10)$ tube as a function of $E^{*}=\hbar \omega$ at two different energies: $\epsilon=0$ (main figure) and $\epsilon=2.0 \mathrm{eV}$ (inset). 
behavior or voltage follows current which leads to capacitive behavior. Specifically, if the imaginary part of $g_{12}(\omega)$ is negative, then the tube displays capacitive behavior. A positive imaginary part implies inductive behavior. Figure 2 indicates that the ac response of a perfect metallic tube oscillates between inductive and capacitive behavior as a function of frequency. Roughly speaking, the dynamic response is inductive for ranges of $\hbar \omega$ where the dissipative part of $g_{12}(\omega)$ is going down from high values, while it is capacitive for ranges where the dissipative part is going up from low values. Thus, as illustrated in Fig. 2, the real and imaginary parts of the dynamic conductance track each other pretty well.

Next, we consider the dynamic conductance of a $(12,0) /$ $(6,6)$ matched electronic heterojunction. The dc conductance of this metal-metal junction has previously been explored [3], and was shown to have a large gap about the Fermi level. Such a behavior clearly demonstrates that the presence of a scattering center can change quantum transport in dramatic ways. The dynamic response of this junction is found to be quite different from that of the dc transport, as illustrated in Fig. 3. Because of photonassisted transport, there is a significant increase in the ac conductance at the Fermi level depending on the ac frequency, almost reaching two conductance quanta at $\hbar \omega=1 \mathrm{eV}$. Hence the metallic behavior of the entire tube is completely restored through a photon-assisted process. Similarly, photon-assisted tunneling allows for current to flow through semiconducting nanotubes, since electrons can now tunnel across the band gap and into the conduction bands. This is illustrated in the inset of Fig. 4, which shows that the ac conductance of a semiconducting $(17,0)$ zigzag tube develops a significant nonzero value under frequencies of $\hbar \omega \approx 0.5 \mathrm{eV}$, or more.

We have also explored the dynamic conductance of tubes of different helicities and with defects. Qualitatively they all display features that are similar to the cases already

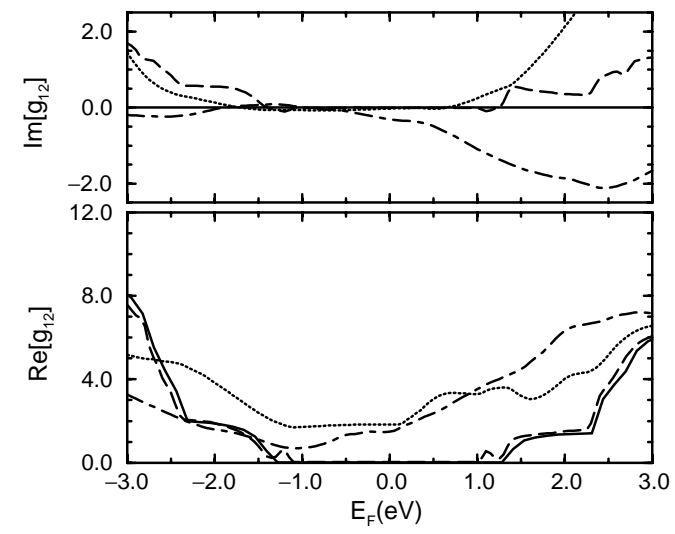

FIG. 3. Real and imaginary component of $g_{12}$ (units $2 e^{2} / h$ ) for a $(12,0) /(6,6)$ metal-metal heterojunction. Note the large gap centered about the Fermi level when frequency is zero. The lines mark the dynamic response at different frequencies: solid line, $\hbar \omega=0 \mathrm{eV}$; dashed line, $0.1 \mathrm{eV}$; dotted line, $1.0 \mathrm{eV}$; and dot-dashed line, $2.0 \mathrm{eV}$. discussed. Figure 4 shows $g_{12}(\omega)$ of a $(10,10)$ tube with a (5-7-7-5) defect in the scattering region. Such a defect forms spontaneously on nanotubes under a large tension via a Stone-Wales transformation. Its energetics is believed to dominate the mechanical properties of the nanotubes [27]. We note that while increased quantum scattering leads to an enhancement in the conductance fluctuations under dc and low-frequency conditions for the tubes with defects, these features are washed out almost entirely for large values of $\hbar \omega$. In contrast to the pristine $(10,10)$ tubes, the initial decrease in the ac conductance as $\hbar \omega$ increases from zero is much weaker, indicating that the presence of a scattering center can also influence ac quantum transport substantially.

These results have, of course, important implications for operation of nanotube-based devices under optical frequency radiation. Photon-assisted transport has been a very useful technique to investigate the electron excitation spectrum of semiconductor quantum dots $[19,28]$. Our results on the dynamic conductance on perfect tubes suggest that one can expect to observe similar photon-assisted resonant transmission in nanotube-based tunnel junctions and nanotube-based quantum dots, which otherwise have a large resistance. Note that the ac frequencies examined in this paper are well in the IR to optical range; hence it is very useful to further explore possibilities of optoelectronic applications when combined with present day semiconductor technology. Finally, it is well known in mesoscopic physics that a bent quantum wire has at least a single quantum bound state localized near the bend [29]. One can therefore expect that similar bound states also exist for bent nanotubes. It will thus be very interesting to explore photonic excitation of these states by experimentally measuring the photocurrent in the ac regime.

In summary, we have investigated the dynamic conductance of carbon nanotubes within the nonequilibrium

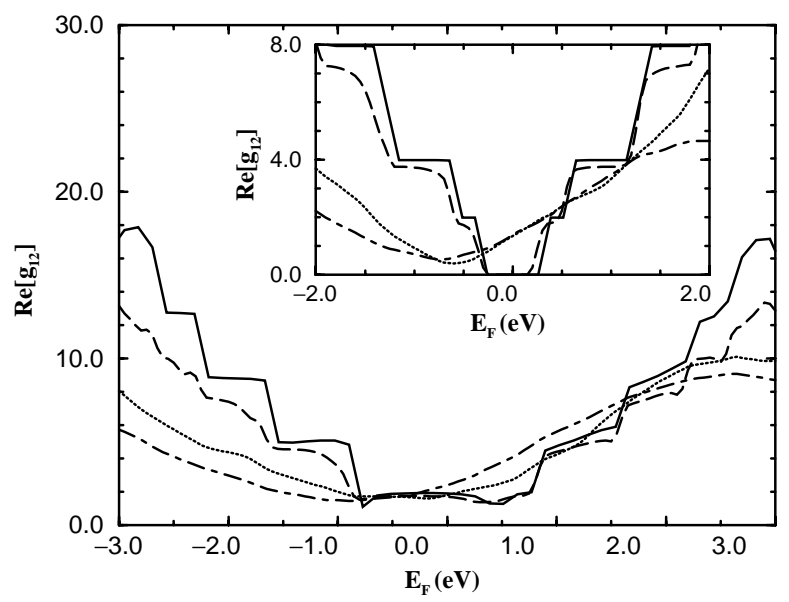

FIG. 4. Real part of $g_{12}$ (units $2 e^{2} / h$ ) for a $(10,10)$ tube with a Stone-Wales rotation, i.e., (5-7-7-5) defect, in the scattering region for different frequencies: solid line, $\hbar \omega=0 \mathrm{eV}$; dashed line, $0.1 \mathrm{eV}$; dotted-line, $1.0 \mathrm{eV}$; and dot-dashed line, $2.0 \mathrm{eV}$. The inset shows the real part of $g_{12}$ for a semiconducting $(17,0)$ zigzag tube. 
Green's function formalism in the context of tight-binding Hamiltonian. We have shown that dynamic conductance through nanotubes differs considerably from the dc case in important ways. Large variations in the response are obtained as a function of the ac frequency. One should be able to probe these variations experimentally using optical radiation on electrically biased nanotubes. The dynamic response is clearly important for device applications, as illustrated by transport through different heterojunctions.

We thank D. Orlikowski for preparing some of the configurations. The following are gratefully acknowledged for financial support: NSERC of Canada and FCAR of Quebec (H. G); RGC grant (HKU 7115/98P) from the Hong Kong SAR (J. W.); and ONR and NASA (C. R.). We also thank the North Carolina Supercomputing Center (NCSC) for extensive computer support.

[1] J. W. Mintmire, B. I. Dunlap, and C. T. White, Phys. Rev. Lett. 68, 631 (1992); N. Hamada, S. I. Sawada, and A. Oshiyama, Phys. Rev. Lett. 68, 1579 (1992); R. Saito, M. Fujita, G. Dresselhaus, and M. S. Dresselhaus, Appl. Phys. Lett. 60, 2204 (1992); Phys. Rev. B 46, 1804 (1992); R. Saito, G. Dresselhaus, and M.S. Dresselhaus, Phys. Rev. B 53, 2044 (1996).

[2] X. Blase, L. X. Benedict, E. L. Shirley, and S. G. Louie, Phys. Rev. Lett. 72, 1878 (1994); Y. A. Krotov, D.-H. Lee, and S. G. Louie, Phys. Rev. Lett. 78, 4245 (1997).

[3] L. Chico, V.H. Crespi, L. X. Benedict, S. G. Louie, and M. L. Cohen, Phys. Rev. Lett. 76, 971 (1996); L. Chico, L. X. Benedict, S. G. Louie, and M. L. Cohen, Phys. Rev. B 54, 2600 (1996); V. H. Crespi, M. L. Cohen, and A. Rubio, Phys. Rev. Lett. 79, 2093 (1997).

[4] L. Chico, M. P. Lopez Sancho, and M.C. Munoz, Phys. Rev. Lett. 81, 1278 (1998).

[5] W. Tian and S. Datta, Phys. Rev. B 49, 5097 (1994).

[6] R. Tamura and M. Tsukada, Phys. Rev. B 55, 4991 (1997); 58, 8120 (1998).

[7] M. P. Anantram and T. R. Govindan, Phys. Rev. B 58, 4882 (1998).

[8] A. A. Farajian, K. Esfarjani, and Y. Kawazoe, Phys. Rev. Lett. 82, 5084 (1999).

[9] M. Buongiorno Nardelli, Phys. Rev. B 60, 7828 (1999); M. Buongiorno Nardelli and J. Bernholc, Phys. Rev. B 60, 16338 (1999).

[10] A. Rochefort, Ph. Avouris, F. Lesage, and D. Salahub, Phys. Rev. B 60, 13824 (1999); A. Rochefort, D. Salahub, and Ph. Avouris, Chem. Phys. Lett. 297, 45 (1998).

[11] J. W. G. Wildoeer, L. C. Venema, A. G. Rinzler, R. E. Smalley, and C. Dekker, Nature (London) 391, 59 (1998); J. W. Odom, J.-L. Huang, P. Kim, and C. M. Lieber, ibid. 391, 62 (1998).

[12] P. G. Collins, Z. Zettl, H. Bando, A. Thess, and R. S. Smalley, Science 278, 100 (1996); S. N. Song, X. K. Wang, R. P. H. Chang, and J. B. Ketterson, Phys. Rev. Lett. 72, 697 (1994); L. Langer, L. Stockman, J. P. Heremans, V. Bayot, C. H. Olk, C. Van Haesendonck, Y. Bruynseraede, and J.-P. Issi, J. Mater. Res. 9, 927 (1994); L. Langer,
V. Bayot, E. Grivei, J.-P. Issi, J. P. Heremans, C. H. Olk, L. Stockman, C. Van Haesendonck, and Y. Bruynseraede, Phys. Rev. Lett. 76, 479 (1996); S. J. Tans, M. H. Devoret, H. Dai, A. Thess, R.E. Smalley, L. J. Georliga, and C. Dekker, Nature (London) 386, 474 (1997); A. Bachtold, C. Strunk, J.-P. Salvetat, J.-M. Bonnard, L. Forro, T. Nussbaumer, and C. Schoenenberger, Nature (London) 397, 673 (1999).

[13] A. Bezryadin, A. R. M. Verschueren, S. J. Tans, and C. Dekker, Phys. Rev. Lett. 80, 4036 (1998).

[14] S. Paulson, M. R. Falvo, N. Snider, A. Helser, T. Hudson, A. Seeger, R. M. Taylor II, R. Superfine, and S. Washburn, http://xxx.lanl.gov/abs/cond-mat/9905304, 1999.

[15] M. Büttiker, Nuovo Cimento Soc. Ital. Fis. 110B, 509 (1995); M. Büttiker, A. Pretre, and H. Thomas, Phys. Rev. Lett. 70, 4114 (1993).

[16] A.P. Jauho, http://xxx.lanl.gov/abs/cond-mat/9911282, 1999.

[17] R. Landauer, IBM J. Res. Dev. 1, 233 (1957); M. Büttiker, Y. Imry, R. Landauer, and S. Pinhas, Phys. Rev. B 31, 6207 (1985).

[18] S. Datta, Electronic Transport in Mesoscopic Systems (Cambridge University Press, Cambridge, 1995).

[19] T. H. Oosterkamp, L.P. Kouwenhoven, A. E. A. Koolen, N. C. van der Vaart, and C. J. Harmans, Phys. Rev. Lett. 78, 1536 (1997).

[20] P. K. Tien and J. R. Gordon, Phys. Rev. 129, 647 (1963); P. S. S. Guimaraes et al., Phys. Rev. Lett. 70, 3792 (1993); B. J. Keay et al., Phys. Rev. Lett. 75, 4098 (1995); 75, 4102 (1995); L. P. Kouwenhoven et al., Phys. Rev. B 50, 2019 (1994); Phys. Rev. Lett. 73, 3443 (1994); R. H. Blick et al., Appl. Phys. Lett. 67, 3924 (1995).

[21] D. Sokolovski, Phys. Rev. B 37, 4201 (1988); C. Bruder and H. Schoeller, Phys. Rev. Lett. 72, 1076 (1994).

[22] B. Wang, J. Wang, and H. Guo, Phys. Rev. Lett. 82, 398 (1999).

[23] The nonequilibrium Green's function approach used here gives identical results to the scattering matrix theory which explicitly solves the combined Poisson's and Schroedinger equations self-consistently, thereby rigorously enforcing the conservation laws. For a more detailed discussion, see B. G. Wang, J. Wang, and H. Guo, J. Appl. Phys. 86, 5094 (1999).

[24] See, for example, N. S. Wingreen, A. P. Jauho, and Y. Meir, Phys. Rev. B 48, 8487 (1993); A. Schiller and S. Hershfield, Phys. Rev. Lett. 77, 1821 (1996).

[25] Examination shows that the inclusion of terms beyond the wideband limit as given in Ref. [22] does not change the results in any significant way. We reserve an examination of these extensions and a study of the high-temperature effects (which essentially smears out the peaks) for a future publication.

[26] T. Pichler et al., Phys. Rev. Lett. 80, 4729 (1998).

[27] M. Buongiorno Nardelli, B. I. Yakobson, and J. Bernholc, Phys. Rev. B 57, R4277 (1998); Phys. Rev. Lett. 81, 4656 (1998).

[28] U. Merkt, Physica (Amsterdam) 189B, 165 (1993); R. Strenz et al., Phys. Rev. Lett. 73, 3022 (1994); K. Brunner et al., Phys. Rev. Lett. 73, 1138 (1994).

[29] Jian Wang and Hong Guo, Appl. Phys. Lett. 60, 654 (1992); 65, 1793 (1994). 